\title{
Hounsfield unit density in the characterisation of bile duct lesions
}

\author{
Abdussamet Batur 1A.B.E.F, Ulku Kerimoglu ${ }^{2 A, C}$, Huseyin Ataseven ${ }^{2 C, D, F}$ \\ 'Selcuk University School of Medicine, Konya, Turkey \\ ${ }^{2}$ Necmettin Erbakan University School of Medicine, Konya, Turkey
}

\section{Abstract}

\begin{abstract}
Purpose: To study the density of lesions in the lumens of extrahepatic bile ducts through computed tomography examinations, and to compare their characterisations with the results of pathology and/or endoscopic retrograde cholangiopancreatography (ERCP).
\end{abstract}

Methods: The density of lesions along extrahepatic bile ducts were measured and compared with pathology and/or ERCP. The lesions were evaluated in two main groups: benign or malignant. The bile duct wall enhancement, intrahepatic bile ducts, and gallbladder were also evaluated.

Results: The study was conducted with computed tomography scans of 197 cases (93 females, 104 males) who had optimal imaging. The results regarding density of extrahepatic intraductal lesions that were studied at BT were compared with pathology and magnetic resonance cholangiopancreatography results. Of 197 lesions, 125 (63.5\%) were reported as benign and $72(36.5 \%)$ were reported as malignant. The results for benign lesions showed an average density of $66.67 \pm 17.30$ Hounsfield units (HU), and for malignant lesions the average density was $82.38 \pm 13.67 \mathrm{HU}$.

Conclusion: Lesion density level (HU) gives valuable information for the differentiation between benign and malign lesions in intraluminal extrahepatic bile ducts.

Key words: Hounsfield unit, CT, bile duct pathologies.

\section{Introduction}

Bile duct pathologies represent a common group of diseases leading to right upper quadrant pain, jaundice, pruritus, nausea, and vomiting. They may be intrahepatically or extrahepatically located. The pathology causing clinical or radiological symptoms may have an intraluminal limitation; moreover, it may originate from the wall or be of extrabiliary system origin, leading to a bile duct disease through pressure and/or invasion.

Ultrasonography (US) and magnetic resonance cholangiopancreatography (MRCP) are the primary diagnostic methods in cases of bile duct diseases. However, computed tomography (CT) provides more detailed information on the obstruction level, mass characterisation, and staging in obstructions due to tumoral lesions. CT is a guideway for demonstrating gallstone, stricture, inflammation, external pressure, intraluminal or extralu- minal tumours, and tumour staging [1,2]. The sensitivity of CT for demonstrating the obstruction level has been reported as $88-97 \%$. This level of sensitivity results from the demonstration of distinctly contrasted hepatic and pancreatic parenchyma and the contrast difference with the biliary system. CT has been reported to have a sensitivity of $100 \%$ for demonstrating biliary obstruction due to malignant infiltration in the intrahepatic or proximal main bile duct [3]. In addition, one of the superiorities of CT is density measurement (Hounsfield units [HU]), which can be used for lesion characterisation; this is not offered by other imaging methods. The present study aimed to perform density measurements of the lesions in the extrahepatic bile duct lumen detected on CT scans performed for biliary system pathology, and to compare the lesion characterisation with the pathology and/or endoscopic retrograde cholangiopancreatography (ERCP) results.

\section{Correspondence address:}

Abdussamet Batur, Selcuk University School of Medicine, Konya, Turkey, e-mail: baturabdussamet@gmail.com

Authors' contribution:

A Study design · B Data collection · C Statistical analysis · D Data interpretation · E Manuscript preparation · F Literature search · G Funds collection 

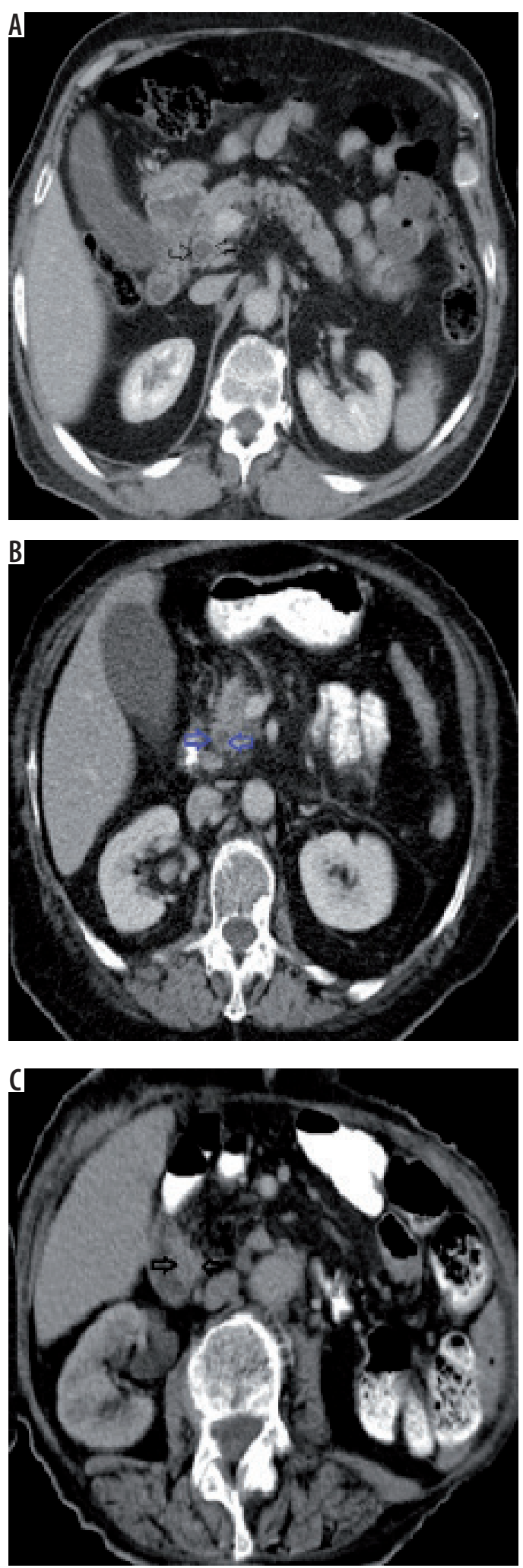

Figure 1. Axial contrast-enhanced computed tomography demonstrates the choledochal wall as (A) thin regular enhancement $(<1.5 \mathrm{~mm})$, (B) thick regular enhancement ( $\geq 1.5 \mathrm{~mm})$, and $(C)$ thick irregular enhancement

\section{Material and methods}

The present study was conducted with the permission and approval of the Medical Ethics Committee dated March 16, 2012 and No. 2012/66. The study evaluated
223 contrast-enhanced CT scans performed for bile duct pathologies between 2009 and 2015. Of these, 17 cases with a density $\geq 150 \mathrm{HU}$ and a clearly known calcification stone and nine cases with a cannula located in bile ducts preventing optimum measurement of lesion density were excluded. Thus, the study included 150 cases from 2009-2013 for the author's thesis, and 47 patients treated in 2013-2015 were added for the article. The study was performed retrospectively.

CT scans were performed with a Siemens Somatom AR Star (Erlanger, Germany). The standard scanning parameters were $130 \mathrm{kVp}$ and $83 \mathrm{~mA}$, and the slice thickness was $5 \mathrm{~mm}$; the reconstruction interval was $4 \mathrm{~mm}$, and the pitch ratio was 1.4 .

After 6-8-hours of fasting in all the patients, $2 \mathrm{ml} / \mathrm{kg}$ of iodised contrast agent was intravenously administered at $4 \mathrm{ml} / \mathrm{s}$ via an automatic injector, including the hepatic dome and the bottom edge of the third part of the duodenum, to obtain contrast-enhanced sections. Non-ionic agents containing $200 \mathrm{mg} / \mathrm{ml}$ of iodine were used as the contrast agent. Images were taken at 70 seconds, the time of the most intensive enhancement for the liver and biliary system at the portal phase following intravenous injection of the contrast agent.

The densities of all the lesions from the portal phase sections were measured as being uninformed of pathology and/or ERCP by using regions of interest (ROIs) via the software on density measurement in the device and HU. The ROI measurement was generally performed at the section with the lesion of the largest size and such that the lesion showed the densest appearance and the largest part of the lesion was covered but the calcification and necrosis areas were not included.

The densities of the lesions in the lumen along the extrahepatic bile ducts were measured and compared with the pathology and/or ERCP. Lesions were evaluated in two main groups: benign and malignant. Along with the lesions, the choledochus diameter, choledochal wall enhancement, intrahepatic biliary tract, and gallbladder were evaluated, and the differences in findings were examined based on the nature of the lesion.

The choledochal wall enhancement was evaluated by four parameters as negative enhancement, thin regular enhancement $(<1.5 \mathrm{~mm})$, thick regular enhancement $(\geq 1.5 \mathrm{~mm}$ ), and irregular enhancement (Figure 1A-C). The median value of the four parameters was taken as a statistical reference. The pancreatic duct diameter was evaluated as either normal or dilated. Moreover, the intrahepatic bile duct was evaluated as either normal or dilated, and the gallbladder transverse diameter $>40 \mathrm{~mm}$ was considered hydropic [4]. The appearances of cases on ERCP and the pathological diagnoses were considered as the gold standard.

The statistical analysis was performed using SPSS 16.0 for Windows software. Descriptive statistics for the studied variables (characteristics) were presented as mean, 
standard deviation, and minimum and maximum values. Student's $t$-test was used for comparing Malign and Benign Group means for the studied variables. For determination of the linear relations among the variables, Pearson's correlation analysis was carried out. The cut-off value of density was determined using receiver operating characteristic (ROC) analysis.

\section{Results}

The study was conducted with CT scans of 223 patients, performed to determine the bile duct pathology. However, 17 cases with a density $\geq 150 \mathrm{HU}$ and a clearly known calcification stone and nine cases with a cannula located in the bile ducts, preventing optimum measurement of lesion density, were excluded from the study. Ultimately, 197 cases who had optimal imaging and could not be diagnosed by the appearance alone were included in the study.

Of the 197 patients included in the study, 93 were female (47.2\%) and 104 were male (52.8\%); the age of all patients ranged from 30 to 94 years (mean age: $65.31 \pm 12.05$ years). The mean ages for male and female participants were $65.34 \pm 12.41$ years and $65.29 \pm 11.78$ years, respectively. The mean ages of occurrence were $65.86 \pm 12.18$ years and $64.38 \pm 11.87$ years for benign and malignant lesions, respectively, and there was no statistically significant difference between age and lesion nature $(p=0.408)$.

Of the 197 lesions with nature detected by histopathology and/or ERCP, 125 (63.5\%) were reported as benign and $72(36.5 \%)$ were reported as malignant. Of the 72 malignant lesions diagnosed by pathology, pancreatic adenocarcinoma was detected in 62, pancreatic acinar cell carcinoma in three, and Klatskin's tumour in seven of the malignant lesions. Besides malignant lesions, some inflammatory pathologies (sclerosing cholangitis, $n=2$ ) and strictures (due to chronic inflammation, $n=11$ ) were also diagnosed by pathology. Of the 125 benign lesions diagnosed by ERCP, except sclerosing cholangitis $(n=2)$ and chronic inflammation $(n=11)$, bile duct stone was detected in 64 patients (mean density: 74.75 with a range of 43-127 HU), bile duct sludge with micro calculus in 39 patients, biliary sludge with added infection in five patients, and hydatid cyst particles in four patients.

Lesion accompanied by contrasted choledochal wall was examined in four categories $(1=$ negative enhancement, $2=$ thin diffuse enhancement $(<1.5 \mathrm{~mm}), 3=$ thick diffuse enhancement $(\geq 1.5 \mathrm{~mm}), 4=$ irregular enhancement). Of the cases, 82 (41.6\%) were category 1, 56 (28.4\%) were category 2, 31 (15.7\%) were category 3 , and 28 (14.2\%) were category 4 . The statistical correlation was performed by taking a median value of " 2 " as a reference. The evaluation towards benign lesions accompanied by contrasting of categories 1 and 2 and malignant lesions accompanied by contrasting of categories 3 and 4 was found to be significant $(p=0.001)$.
Dilatation in intrahepatic bile ducts was observed in 95 (76\%) of 125 benign cases and 66 (91.7\%) of 72 malignant cases. Intrahepatic bile ducts were at normal width in $24 \%(n=30)$ of benign lesions and $8.3 \%(n=6)$ of malignant cases. There was a statistically significant correlation between malignancy and the presence of dilatation in the intrahepatic bile ducts $(p=0.016)$.

The gallbladder pathologies accompanied by lesion nature in extrahepatic bile ducts were evaluated. Forty-eight $(24.4 \%)$ patients were cholecystectomised. There was no hydrops in $84(42.7 \%)$ of the cases. Of patients with hydropic gallbladder $(n=65), 30(46.2 \%)$ had benign lesions and 35 (53.8\%) had malignant lesions in choledochal lumen. A statistical significance was found between the gallbladder hydrops and the nature of lesion located in extrahepatic bile ducts $(p=0.009)$.

The results from comparing CT measurements of the densities of extrahepatic intraductal lesions with the pathology and/or ERCP findings revealed that the mean density was $66.67 \pm 17.30 \mathrm{HU}$ in benign cases $(n=125)$ (Figure 2) and $82.38 \pm 13.67 \mathrm{HU}$ in malignant cases $(n=72$ ) (Figure 3). The ROC curve for distinguishing benign lesions from malignant lesions is shown in Figure 4.

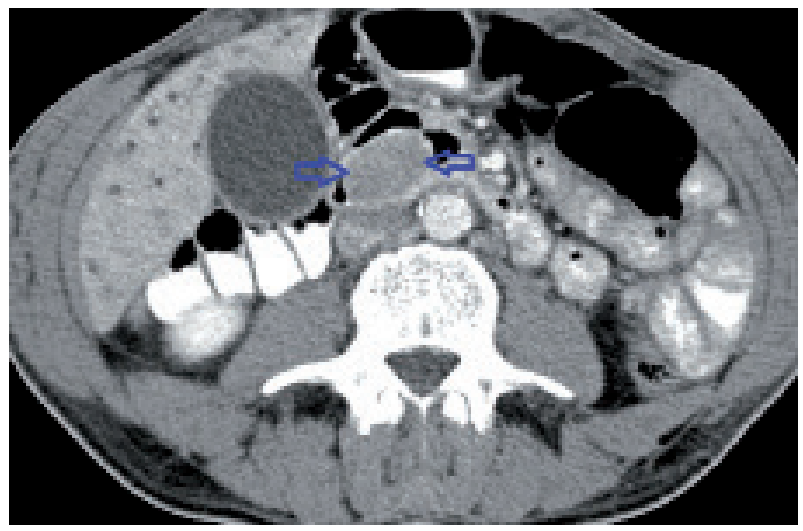

Figure 2. Axial contrast-enhanced computed tomography demonstrates bile duct calculi with a density of $72 \mathrm{HU}$

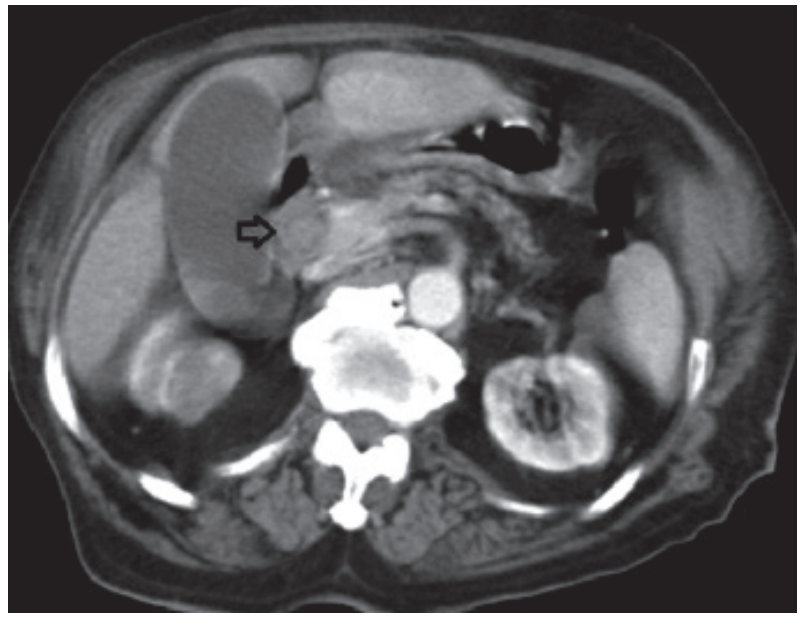

Figure 3. Axial contrast-enhanced computed tomography demonstrates adenocarcinoma filling the lumen of the bile duct with a density of $86 \mathrm{HU}$ 


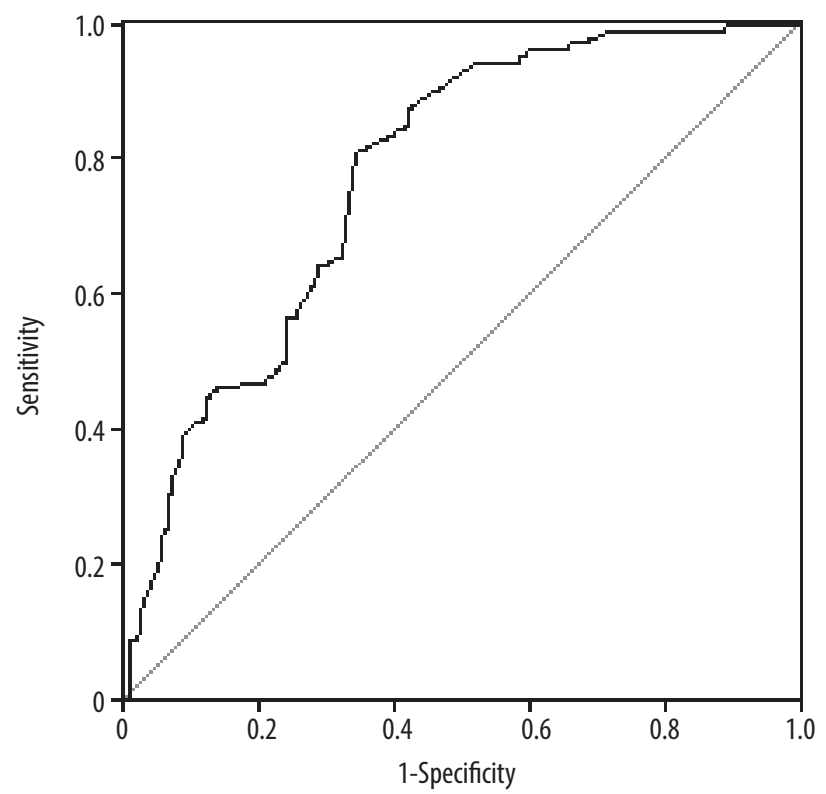

Figure 4. The area under the receiver operating characteristic (ROC) curve for distinguishing benign lesions from malignant lesions using $\mathrm{HU}$ was 0.774 (95\% Cl: $0.710-0.839, p=0.001)$

A cut-off value for lesion density greater than $73.5 \mathrm{HU}$ yielded sensitivity and specificity values of $80.6 \%$ and $65.6 \%$, respectively, for differentiation of benign and malignant extrahepatic biliary tract lesions $(p=0.001)$. The intraclass correlation coefficient for density measurement was $77.4 \%$ (95\% CI: 0.710-0.839).

\section{Discussion}

Radiological imaging methods have great importance for evaluating the bile ducts. The use of CT to evaluate the bile ducts is not common; however, its use is becoming more common due to the high image resolution and fast scanning option as well as the availability of multisection CT and thereby the ability to reconstruct the images and to obtain coronal-sagittal images. It was reported that the localisation of obstructive pathology could be detected at $88-97 \%[3,5]$.

There are studies that have reported about the association of choledochal wall thickness and the wall contour characteristics with malignancy. The study by Schulte et al. reported that a thickness $\geq 1.5 \mathrm{~mm}$ was abnormal and a choledochal wall thickness $\geq 5 \mathrm{~mm}$ was seen only in cholangiocarcinoma. The comparative analysis of the choledochal wall enhancement and the pancreas head enhancement in cases with and without bile duct pathology revealed increased levels of enhancement in cases with lesions [6]. In the same study, it was demonstrated that wall enhancement was possible also without any lesions in the bile ducts. Therefore, wall enhancement alone does not indicate the presence of pathology. The present study could not evaluate wall enhancement in patients with no lesions because only patients with lesions in bile ducts were included. The stricture and wall thickness due to benign lesions were regular and symmetrical [7]. An irregular, asymmetrical wall thickness is generally seen in malignant lesions $[8,9]$. Park et al. [10] reported that the irregular margin and asymmetrical luminal shrinking could also be present in benign lesions at a rate of $26 \%$ and $35 \%$, respectively. The present study $(n=197)$ examined 138 cases with fine-regular enhancement and negative enhancement with a thickness of $<1.5 \mathrm{~mm}$ and 59 cases with thick-regular or irregular enhancement with a thickness of $\geq 1.5 \mathrm{~mm}$. The wall thickness and enhancement pattern was of malignant character in 43 (59.72\%) of the malignant lesions $(n=72)$ and $16(12.8 \%)$ of the benign lesions $(n=125)$. Malignancy was detected in $13(15.85 \%)$ of 82 cases with no contrasted wall. The association between the choledochus thickness and the wall contour characteristics with benign-malignant nature was found to be statistically significant $(p=0.001)$.

In a study on intrahepatic bile ducts, a distinct dilatation was reported in $69 \%$ of malignant lesions and $28 \%$ of benign lesions [1]. It was reported that there was no dilatation in approximately $1 / 3$ of patients with benign lesions, whereas all malignant cases had dilatation. In our study, there was no dilatation of intrahepatic bile ducts in only six (8.3\%) of 72 malignant cases, whereas the intrahepatic bile ducts were at normal width in 30 (24\%) of 125 benign lesions.

In a study on gallbladder hydrops in patients with choledochal lesion $(n=50)$, there was a malignant lesion in the distal of choledochus in 11 of 12 cases with hydrops, and a pericholecystic abscess pressuring on cystic duct was observed in one case as a benign lesion [1]. In the present study, there was hydrops in $30(46.2 \%)$ of these patients had benign lesions, and 35 (53.8\%) had malignant lesions in choledochal lumen. It was statistically confirmed that the malignant extrahepatic bile duct lesions are accompanied by gallbladder hydrops $(p=0.009)$.

Biliary obstruction is most commonly due to choledocholithiasis; however, it may also occur due to other pathologies [7]. Stones located in bile ducts may not always lead to dilatation. The sensitivity of CT is low in undilated bile ducts and low-density stones [11]. The sensitivity of CT varies from $72 \%$ to $88 \%$ for detecting choledochus stones [8]. Its stone attenuation varies according to the content. Hickmann et al. reported that a density of $>50 \mathrm{HU}$ indicated bilirubin content and a density of $<50 \mathrm{HU}$ indicated cholesterol content [12]. In the present study, stone was detected in 64 (42.95\%) cases and the stone content-density association was excluded from the study scope.

We could not reach any resource information on measuring the density of bile duct malignancies in the literature; however, it has been reported that the bile duct density might vary considerably depending on the content $[8,13,14]$. Based on our research, there is also no literature information about the variability of the $\mathrm{HU}$ attenuation 
value of bile duct lesions by lesion nature. Therefore, it is not possible for us to compare the data obtained in the present study with the data of other studies.

In the present study, the mean $\mathrm{HU}$ attenuation value on CT was $66.67 \pm 17.30 \mathrm{HU}$ for benign lesions $(n=125)$ and $82.38 \pm 13.67 \mathrm{HU}$ for malignant lesions $(n=72)$, detected in the bile ducts. A cut-off value greater than $73.5 \mathrm{HU}$ yielded sensitivity and specificity values of $80.6 \%$ and $65.6 \%$, respectively, for differentiation of intraductal malignant lesions from benign lesions. The intraclass correlation coefficient for HU measurement was $77.4 \%$ (95\% CI: 0.710-0.839). When the "cut-off" value was taken as 73.5 , it was found statistically significant that the lesions of higher density could be interpreted in favour of malignancy and the lesions of lower density could be interpreted in favour of benignity $(p=0.001)$.

\section{Conclusions}

Although CT is not the main examination method for lesions located in the bile ducts, it provides highly beneficial information for determining the obstruction level in the presence of bile duct dilatation, detecting the intraluminal and extraluminal lesions, demonstrating the calcification, and identifying the accompanying hepatic and biliary lesions.

Direct and indirect findings are guidance for the detection of the nature of intraluminal lesions. Among the indirect findings, intrahepatic bile duct dilatation, gallbladder hydrops is most common in malignant lesions. Among the direct findings, thick and irregular enhancement of the choledochal wall adjacent to the lesion and a wall thickness $>4 \mathrm{~mm}$ should be suggestive of malignant aetiology. It has been seen that lesions with a density $>73.5$ HU have a higher possibility of malignancy, except for the lesions with diffuse calcification (calculus).

\section{Disclosure}

The authors declare no conflict of interests.

\section{References}

1. Baron RL, Stanley RJ, Lee JKT, Koehler RE, Levitt RG. Computed tomography features of biliary obstruction. Am J Roentgenol 1983; 140: 1173-1178.

2. Kamel IR, Liapi E, Fishman EK. Liver and biliary system: evaluation by multidetector CT. Radiol Clin N Am 2005; 43: 977-997.

3. Wyatt SH, Fishman EK. Biliary tract obstruction: the role of spiral CT in detection and definition of disease. Clin Imaging 1997; 21: 27-34.

4. Prokop M, Galanski M. Spiral and Multislice Computed Tomography of the Body. Thieme, Stuttgart, New York 2003. p. 477-540

5. Anderson SW, Lucey BC, Varghese JC, Soto JA. Accuracy of MDCT in the diagnosis of choledocholithiasis. AJR Am J Roentgenol 2006; 187: 174-180.

6. Schulte SJ, Baron RL, Teefey SA, Rohrmann CA, Freeny PC, Shuman WP, et al. CT of the extrahepatic bile ducts: wall thickness and contrast enhancement in normal and abnormal ducts. AJR Am J Roentgenol 1990; 154: 79-85.

7. O'Connor OJ, O'Neil S, Maher MM. Imaging of biliary tract disease. AJR Am J Roentgenol 2011; 197: 551-558.

8. Yeh BM, Liu PS, Soto JA, Corvera CA, Hussain HK. MR imaging and CT of the biliary tract. Radiographics 2009; 29: 1669-1688.

9. Lim JH. Cholangiocarcinoma: morphologic classification according to growth pattern and imaging findings. AJR Am J Roentgenol 2003; 181: 819-827.

10. Park MS, Kim TK, Kim KW, et al. Differentiation of extrahepatic bile duct cholangiocarcinoma from benign stricture: findings at MRCP versus ERCP. Radiology 2004; 233: 234-240.

11. Giadas TC, De Toledo LSO, Asensio MTMB, et al. Helical CT cholangiography in the evaluation of the biliary tract: application to the diagnosis of choledocholithiasis. Abdom Imaging 2002; 27 : 61-70.

12. Hickmann MS, Sweisinger WH, Bova JD, Kurtin WE. Computer tomographic analysis of gallstones. Arch Surg 1986; 121: 289-291.

13. Bova JG, Schwesinger WH, Kurtin WE. In vivo analysis of gallstone composition by computed tomography. Gastrointest Radiol 1992; 17: 253-256.

14. Janowitz P, Zoller A, Swobodnik W, Wechsler JG, Schumacher KA, Ditschuneit H. Computed tomography evaluation of radiolucent gallstones in vivo. Gastrointest Radiol 1990; 15: 58-60. 